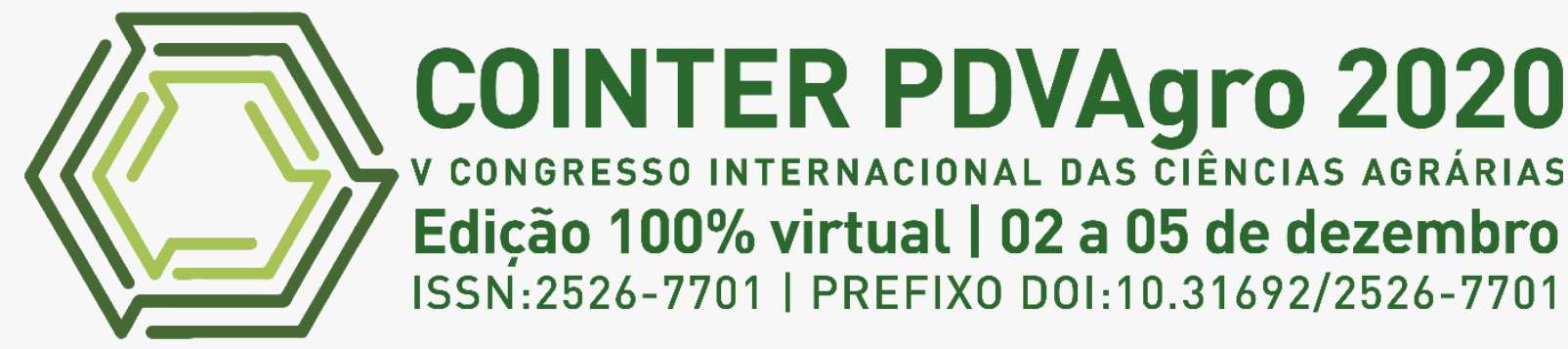

\title{
BALANÇO HÍDRICO CLIMATOLÓGICO MENSAL PARA O MUNICÍPIO DE CASTANHAL - PA EM EVENTOS DE ENOS E EFEITOS NA PRODUÇÃO DO AÇAIZEIRO
}

\section{BALANCE HÍDRICO CLIMÁTICO MENSUAL PARA EL MUNICIPIO DE CASTANHAL - PA EN ENOS EVENTOS DE PRODUCCIÓN AÇAIZEIRO \\ MONTHLY CLIMATE WATER BALANCE FOR THE MUNICIPALITY OF CASTANHAL - PA IN ENOS EVENTS IN AÇAIZEIRO PRODUCTION}

\author{
Apresentação: Comunicação Oral \\ Igor Cristian de Oliveira Vieira ${ }^{1}$; Stefany de Andrade Silva ${ }^{2}$; Saulo de Jesus Dantas ${ }^{3}$ João Carlos dos Santos \\ Duarte $^{4}$; Valter Barbosa dos Santos ${ }^{5}$
}

DOI: https://doi.org/10.31692/2526-7701.VCOINTERPDVAgro.0186

\begin{abstract}
RESUMO
O balanço hídrico climatológico (BHS) é uma importante ferramenta para o planejamento agrícola, fornecendo dados sobre o regíme hídrico da região e a ocorrência de El Niño ou La Niña provoca alterações no clima e ocasiona efeitos diretos na produção das culturas, entre elas, a do açaizeiro que possui importante papel socioeconômico. Portanto, objetivo de trabalho é realizar o balanço hídrico de Castanhal - PA em anos de ENOS e na climatologia e verificar influências na produção do açaizeiro. Para realização do balanço hídrico foi utilizado 30 anos de dados meteorológicos mensais da NASA/POWER e as informações sobre a ocorrencia dos ENOS foram obtidas no site do NOOA. balanço hídrico proposto por Thornthwaite e Mather (1955) foi feito utilizando linguagem de programação Python versão 3.6 e uma capacidade de água disponível (CAD) de $100 \mathrm{~mm}$, geralmente adotada para cultura perenes como o açaizeiro. O BHS disponibilizou informações mais detalhadas do comportamento da T, P, ETp, ETr, Def e Exc ao longo dos anos na ocorrência de El Niño, La Niña em relação a climatologia.As temperaturas apresentaram médias sempre superiores no ano de El Niño em torno de $27,31^{\circ} \mathrm{C}( \pm 1,07)$ em comparação ao ano de La Niña $26,43^{\circ} \mathrm{C}( \pm 0,95)$ e da climatologia $26,39^{\circ} \mathrm{C}$ $( \pm 0,80)$, o mês de março apresentou maior volume de chuva (El Niño $=374,2 \mathrm{~mm}$; La Niña $=525,0$ mm; Clim. $=454,6 \mathrm{~mm}$ ) e o de outubro mais seco (El Niño $=33,9 \mathrm{~mm}$; La Niña $=95,9 \mathrm{~mm}$; Clim. $=$ $60,03 \mathrm{~mm}$ ). A evapotranspiração potencial da região é superior à precipitação pluviométrica de agosto a novembro, resultando em déficit hídrico neste período, sendo intensificado no ano de El Niño. A La Niña no ano de 2011 aumentou exponencialmente a produção de açaí, e de El Niño em 2016 diminui. As disponibilidades de água em Castanhal no período de janeiro a julho apresentaram grande volume de chuva e excedente hídrico, resultando em maiores produções independente da ocorrência ou não de ENOS. Já no período de estiagem (Agosto a Novembro) é recomendada a utilização da irrigação para

\footnotetext{
${ }^{1}$ Mestrando em Agronomia (Ciência do Solos), UNESP - Jaboticabal/SP, igor.vieira@unesp.br

${ }^{2}$ Graduanda em Engenharia Florestal, UFRA - Belém/PA, Stef.engflorestal@gmail.com

3 Mestrando em Agronomia, (Genética e Melhoramento de Plantas) UNESP - Jaboticabal/SP, saulodantas@live.com

${ }^{4}$ Mestrando em Agronomia (Ciência do Solos), UNESP - Jaboticabal/SP, duarte.joaocarlos17@gmail.com

${ }^{5}$ Msc. em Agronomia, valterbarbosa santos@ hotmail.com
} 
suprir a demanda hídrica da cultura, principalmente nos anos de El Niño, onde os resultados apontaram maior déficit e diminuição na produção. Os resultados deste estudo forneceram um suporte para o planejamento agrícola para o açaizeiro no município de Castanhal - PA.

Palavras-Chave: deficiência e excedente hídrico, Euterpe oleracea, El Niño e La Niña.

\title{
RESUMEN
}

El balance hídrico climatológico (BHS) es una herramienta importante para la planificación agrícola, ya que proporciona datos sobre el régimen hídrico de la región y la ocurrencia de El Niño o La Niña provoca cambios en el clima y causa efectos directos en la producción de cultivos, incluyendo del açaizeiro que tiene un papel socioeconómico importante. Por lo tanto, el objetivo de este trabajo es realizar el balance hídrico de Castanhal - PA en años de ENOS y en climatología y verificar influências en la producción de açaizeiro. Para realizar el balance hídrico, 30 años de datos meteorológicos mensuales NASA / POWER e información sobre la ocurrencia de ENOS se obtuvo en el sitio web de NOOA. El balance hídrico propuesto por Thornthwaite y Mather (1955) se realizó utilizando el lenguaje de programación Python versión 3.6 y una capacidad de agua disponible (CAD) de $100 \mathrm{~mm}$, generalmente adoptada para cultivos perennes como el açaí. El BHS brindó información más detallada sobre el comportamiento de T, P, ETp, ETr, Def y Exc a lo largo de los años en la ocurrencia de El Niño, La Niña en relación a la climatología. Las temperaturas presentaron promedios siempre superiores en el año de El Niño alrededor de de $27,31^{\circ} \mathrm{C}( \pm 1,07)$ respecto al año de La Niña $26,43{ }^{\circ} \mathrm{C}( \pm 0,95)$ y climatología $26,39^{\circ} \mathrm{C}( \pm 0,80)$, el mes de marzo presentó mayor volumen de lluvia (El Niño $=374.2 \mathrm{~mm}$; La Niña $=525.0 \mathrm{~mm}$; Clim. $=454.6 \mathrm{~mm}$ ) y el octubre más seco $($ El Niño $=33.9 \mathrm{~mm}$; La Niña $=95.9 \mathrm{~mm}$; Escalada $=60,03 \mathrm{~mm}$ ). La evapotranspiración potencial en la región es mayor que la precipitación de agosto a noviembre, lo que resulta en un déficit hídrico en este período, intensificándose en el año de El Niño. La Niña en 2011 aumentó exponencialmente la producción de açaí y El Niño en 2016 disminuyó. La disponibilidad de agua en Castanhal en el período de enero a julio mostró un alto volumen de lluvia y excedente de agua, lo que resultó en una mayor producción independientemente de la ocurrencia o no de ENOS. En la época seca (agosto a noviembre), se recomienda utilizar el riego para suplir la demanda de agua del cultivo, especialmente en los años de El Niño, donde los resultados mostraron un mayor déficit y disminución de la producción. Los resultados de este estudio apoyaron la planificación agrícola del açaí en el municipio de Castanhal - PA.

Palabras Clave: deficiencia y excedente de agua, Euterpe oleracea, El Niño e La Niña

\begin{abstract}
The climatological water balance (BHS) is an important tool for agricultural planning, providing data on the region's water regime and the occurrence of El Niño or La Niña causes changes in the climate and causes direct effects on crop production, including of the açaizeiro that has an important socioeconomic role. Therefore, the objective of this work is to carry out the water balance of Castanhal - PA in years of ENOS and in climatology and to verify influences on the production of açaizeiro. To carry out the water balance, 30 years of NASA / POWER monthly meteorological data and information on the occurrence of ENOS was obtained on the NOOA website. water balance proposed by Thornthwaite and Mather (1955) was made using Python programming language version 3.6 and an available water capacity (CAD) of $100 \mathrm{~mm}$, generally adopted for perennial crops such as açaí. The BHS provided more detailed information on the behavior of T, P, ETp, ETr, Def and Exc over the years in the occurrence of El Niño, La Niña in relation to climatology. Temperatures presented averages always higher in the year of El Niño around of $27.31^{\circ} \mathrm{C}( \pm 1.07)$ compared to the year of La Niña 26.43 ${ }^{\circ} \mathrm{C}( \pm 0.95)$ and climatology $26.39^{\circ} \mathrm{C}( \pm 0.80)$, the month of March presented higher volume of rain $($ El Niño $=374.2 \mathrm{~mm}$; La Niña $=525.0 \mathrm{~mm} ;$ Clim. $=454.6 \mathrm{~mm})$ and the driest October $($ El Niño $=33.9$ $\mathrm{mm}$; La Niña $=95.9 \mathrm{~mm}$; Clim. $=60.03 \mathrm{~mm}$ ). The potential evapotranspiration in the region is greater than the rainfall from August to November, resulting in a water deficit in this period, being intensified in the year of El Niño. La Niña in 2011 increased the production of açaí exponentially, and El Niño in 2016 decreased ,. The availability of water in Castanhal in the period from January to July showed a high volume of rain and water surplus, resulting in greater production regardless of the occurrence or not of ENOS. During the dry season (August to November), it is recommended to use irrigation to supply the water demand of the crop, especially in the years of El Niño, where the results showed a greater
\end{abstract}


deficit and decreased production. The results of this study provided support for agricultural planning for açaí in the municipality of Castanhal - PA.

Keywords: deficiencia y excedente de agua, Euterpe oleracea, El Niño e La Niña

\section{INTRODUÇÃO}

As culturas sofrem influência direta das alterações do clima e expressam esses efeitos na sua produção, podendo externar de forma positiva ou negativa com altas ou baixas produções nos anos de maiores ou menores índices pluviométricos (PEREIRA et al. 2016) sendo um fator importante principalmente na região amazônica que possui uma carência de estudos relacionados a influência de eventos climáticos nas produções das culturas da região.

A compreensão a cerca do regime hídrico é necessário para um melhor planejamento agrícola e o cálculo do Balanço Hídrico Climatológico serve como uma ferramenta de apoio na montagem das estratégias agrícolas fornecendo dados sobre o regíme hídrico da região. (COUTINHO et al., 2015).O balanço hídrico climatológico é uma técnica que calcula a disponibilidade de água para as culturas contabilizando a evapotranspiração e precipitação com base na capacidade de amarzenamento de água no solo (BARRETO et al., 2009).

O balanço hídrico é o primeiro passo para a dterminação das entradas e saídas de água no solo de uma região, para que se possa estabelecer os períodos de estiagem (deficiência hídrica) e de maiores precipitações (excedente hídrico) (REICHARDT, 1990) fornecendo assim informações que possibilitarão indentificar as áreas onde as culturas podem expressar seu melhor desempenho com maiores produções (BARRETO et al., 2009).

O fenômeno El Niño-Oscilação Sul (ENOS) é um processo acoplado relacionado a fatores de natureza oceânica (El Niño ou La Niña), com influência atmosférica da Oscilação Sul ocasionado aumento ou reduação das chuvas em diversas regiões do planeta. $\mathrm{O}$ açaizeiro (Euterpe oleracea) palmeira nativa das áreas de várzeas dos rios amazônicos (SILVESTRE et al., 2016) possui dupla utilidade econômica, com obtenção de frutos e palmito (YOKOMIZO; FARIAS NETO; OLIVEIRA, 2016). Portanto, objetivo de trabalho é realizar o balanço hídrico de Castanhal - PA em anos de ENOS e na climatologia e verificar influências na produção do açaizeiro.

\section{FUNDAMENTAÇÃO TEÓRICA}

O estado do Pará possui um grande potencial para a produção de açaí que possibilita suprir a demanda crescente pelo produto, destacando-se no cenário nacional como maior consumidor e principal produtor regional e nacional (YOKOMIZO; FARIAS NETO; 
BALANÇO HÍDRICO CLIMATOLÓGICO MENSAL

OLIVEIRA, 2016) correspondendo a cerca de mais de 95\% da produção do país com uma produção de 1.439.249 toneladas no ano de 2017, em uma área colhida de 190.567 hectares, com um rendimento médio de $7.552(\mathrm{~kg} / \mathrm{ha})$, segundo dados elaborados pela Secretaria Estadual de Desenvolvimento Agropecuário e da Pesca (SEDAP, 2018).

A demanda crescente não somente interna, mas externa pelo açai gerou uma transformação na comercilaização do fruto, e no seu modo de obtenção, passando de inteiramente extrativista, onde na busca de maiores produtividades, se realizava o manejo dos acaizais para a produção em terra firme, devido a produção insuficiente das áreas de várzea para suprir o necessidade do mercado (NOGUEIRA; SANTANA; GARCIA, 2013).

Mesmo diante de grande procura pelo mercado nacional e internacional, com base nos dados da Secretaria de Desenvolvimento Agropecuário e da Pesca (SEDAP), apenas cerca de $10 \%$ de toda produção do estado é comercializada para outros países, demonstrando um amplo mercado de expansão, necessitando aumentar a produção para suprir as demandas locais e nacionais que representam cerca de $60 \%$ e $30 \%$ respectivamente, e alcançar os mercados externas de forma mais efetiva (PESSOA; TEXEIRA, 2012; TAVARES; HOMMA, 2015).

A utilização dos produtos do açaizeiro não se resumo somente na utilização do fruto para extração da polpa, o seu uso é praticamente integral, servindo de matéria prima para a confecção de inúmeros subprodutos, demonstranto o seu importante papel socioeconômico (HOMMA et al., 2006) compondo aproximandamente 70\% da renda mensal das populações, principalmente as ribeirinhas (CONAB, 2015).

A partir do açaizeiro são gerados empregos e renda com a sua utilização no paisagismo como planta ornamental, construção de móveis e imóveis, na elaboração de produtos farmacêuticos como vermífugos e antidiarreicos, na produção de celulose (papel Kraft),artesanatos (biojóias), fonte energética (carvão vegetal), adubação orgânica e demais subprodutos oriundos da planta (OLIVEIRA; FARIAS NETO; QUEIROZ, 2014).

$\mathrm{O}$ açaizeiro possui ampla ocorrencia em regiões de clima quente e úmido por ser nativo da região amazônica, estando presente em locais com climas do tipo Af, Am e Aw, com médias de temperatura anuais em torno de $26^{\circ} \mathrm{C}$ e volume de chuvas anuais de aproximadamente de $2.096 \mathrm{~mm}$ (ALVARES et al.;2013). As informações sobre o comportamento do clima de uma região, torna-se essencial para um boa gestão dos recursos hídricos e da própria cultura, auxiliando assim, nas tomadas de decisões no campo (OLIVEIRA;OLIVEIRA, 2018).

A distribuição da produção ocorre de maneira desigual ao longo do ano, possuindo duas safras, a do período chuvoso e seco, onde a primeira ocorre entre os meses de janeiro a julho, 
que representa os meses de maior volume de chuvas e período de florescimento do açaizeiro, no qual caracteriza a entressafra com frutos que produzem uma polpa com qualidade inferior e de cor roxo-azulada. A segunda safra, que ocorre no verão entre os meses de agosto a dezembro, corresponde o período de maior oferta do fruto, representando a sua principal safra, com frutos de qualidade superio que resulta em polpa de coloração vermelho arroxeada (GUIMARÃES, 1998).

Os valores de comercialização na entressafra sofrem grande variação, com preços que quadruplicam, gerando aumento no valor final do produto ao consumidor, e juntamente com a entressafra, esse valor sofre inflência da crescente exportação dessa matéria prima, tais fatores fazem com que a lata do fruto de $14 \mathrm{~kg}$ sofra um aumento de $350 \%$, passando de $\mathrm{R} \$ 50,00$ na safra para ser comercializado por R $\$ 300,00$ na entressafra em alguns estados, obedecendo a lei da oferta e demanda (ARMANDO, 2015)

Um ferramenta que auxilia nesse planejamento climático é o Balanço Hídrico Climatológico Sequencial que trás informações sobre a necessidade e disponibilidade de água no solo de uma região, evidenciando os períodos críticos e demonstrando também os efeitos de eventos climáticos extremos como a ocorrencias de El Niño e La Niña, possibilitando a elaboração de uma melhor estratégia para a cultura de interesse (OLIVEIRA;OLIVEIRA, 2018).

O fenômeno El Niño-Oscilação Sul (ENOS) trata-se de um processo acoplado que envolve além dos fatores de natureza oceânica (El Niño ou La Niña), a inluência do fator atmosférico da Oscilação Sul, que tem como principal característica a variação de pressão atmosférica (ASSAD; PINTO, 2008) promovendo efeitos que ocasionam alterações climáticas em todo país e outros países principalmente em regiões costeiras, aumentando a índice pluviométrico em algumas partes do planeta e provocando estiagens em outras (FIORIN, 2015).

A intensificão de estudos relacionados a compreender as variáveis climáticas na área agrícola tornam-se relevantes para o planejamento agrícola, em virtude do grande número de perdas nesse setor que chega em torno de de noventa e cinco por cento das perdas, relacionadas diretamente a fenômenos de enchentes ou estiagens (ASSAD ; PINTO, 2008).

Essas oscilações no clima ao longos dos anos influenciam no comportamento das culturas e na sua resposta final na produção, como no estudo de Pereira et al. (2016) com a cana de açúcar, que observou a correlação direta das altas ou baixas produções com os volumes de chuva nos anos, demonstrando a importancia de estudos com diferentes culturas, principalente na região amazônica com culturas ainda poucos estudadas como a do açaí. 


\section{METODOLOGIA}

O município de Castanhal pertence a microrregião de Castanhal $\left(1^{\circ} 17^{\prime} 50 " \mathrm{~S}\right.$ e 4755'20"W) (Fig. 1) e segundo a classificação de Koppen o clima da região é do tipo Am com média anual de temperatura de $26{ }^{\circ} \mathrm{C}$, máxima e mínima de 28 e $22{ }^{\circ} \mathrm{C}$ respectivamente, valores de umidade relativa do ar que variam entre, 95 e $79 \%$ e média anual de precipitação em torno de $2500 \mathrm{~mm}$ (ALVARES et al. 2013).

Figura 01. Mapa de localização do município de Castanhal - PA.

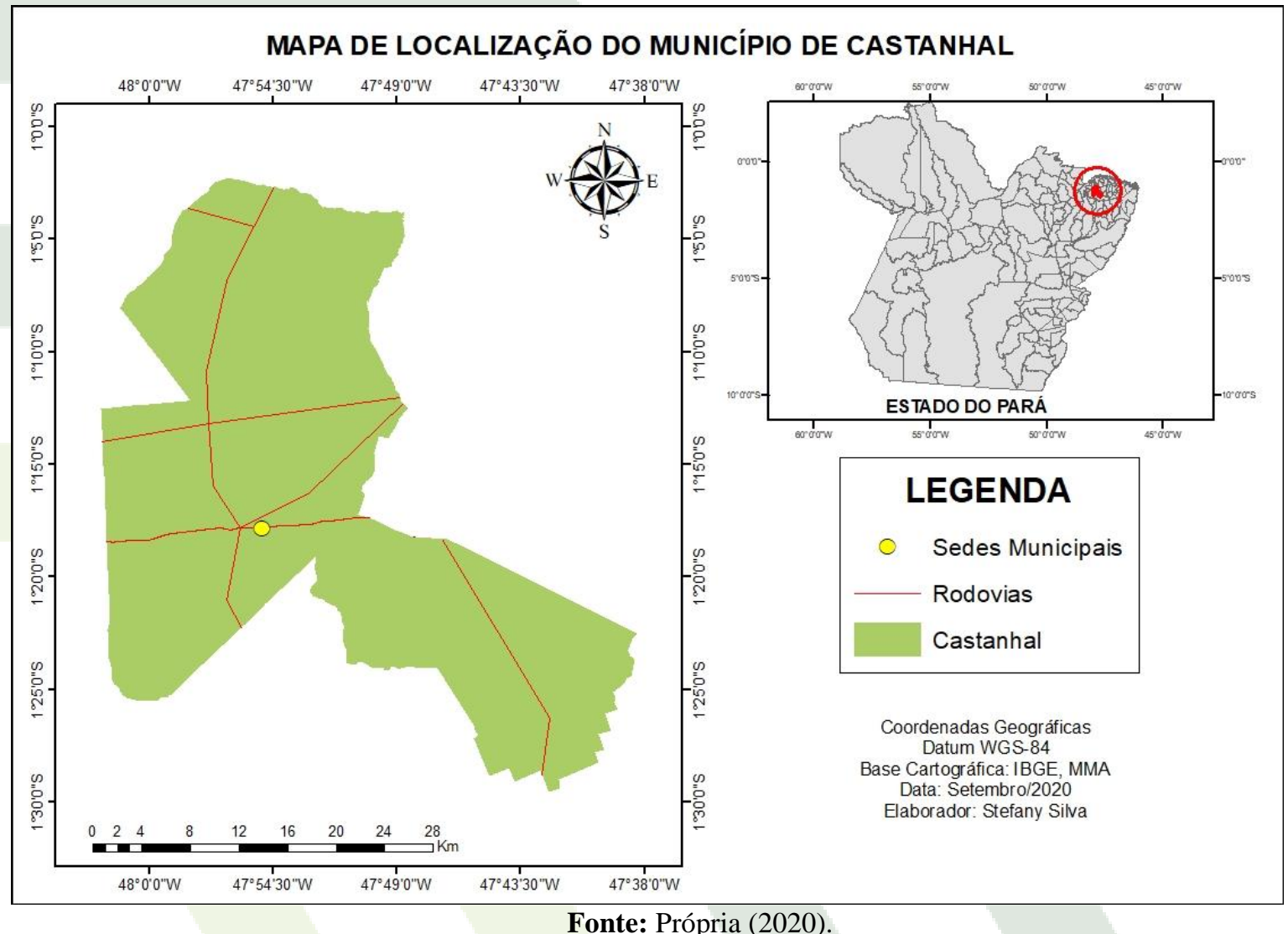

Fonte: Própria (2020).

A obtenção dos dados meteorológicos mensais de temperaturas médias do ar e precipitações para a determinação do balanço hídrico foi realizada através da da plataforma National Aeronautics And Space Administration/Predction of World Wide Enerdy Resources - (NASA/POWER; http://power.larc.nasa.gov), do ano de 1989 até o ano de 2019, totalizando 30 anos. Essa plataforma governamental possui grids com resolução espacial de $0.25^{\circ}$ (latitude-longitude), fornecendo dados meteorológicos para todo mundo (STACKHOUSE et al., 2018).

As informações sobre a ocorrencia dos ENOS foram obtidas por meio dos dados disponibilizados pela National Oceanic and Atmospheric Administration (NOAA). balanço hídrico proposto por Thornthwaite e Mather (1955) foi feito utilizando linguagem de 
programação

Python

versão

3.6.

A

determinação da Evapotranspiração Potencial (ETP) pelo método de Camargo (1971). Foi utilizada uma capacidade de água disponível (CAD) de $100 \mathrm{~mm}$, geralmente adotada para cultura perenes como o açaizeiro (PEREIRA; ANGELOCCI; SENTELHAS, 2007).

\section{RESULTADOS E DISCUSSÃO}

Ao analisar os valores de precipitação e temperatura dos anos de ocorrência de ENOS e da climatologia da região de estudo, observou-se uma redução de 57,92\% da precipitação no ano de El Niño em relação ao ano de ocorrência de La Niña, e diminuição de 38,14\% em comparação com a climatologia (Figura 02).

Segundo Menezes et al. (2015) o município está localizado em uma região de maior pluviosidade do Estado, possuindo um período chuvoso bem definido que compreende os meses de fevereiro, março e abril, tendo como destaque o mês de março com o mais chuvoso (El Niño $=374,2 \mathrm{~mm}$; La Niña $=525,0 \mathrm{~mm}$; Clim. $=454,6 \mathrm{~mm}$ ), e um período de estiagem que se concentram nos meses de setembro, outubro e novembro, tendo como o mês de outubro com o menor volume de chuva $($ El Niño $=33,9 \mathrm{~mm}$; La Niña = 95,9 mm; Clim. = 60,03 mm), dados corroborados no estudo (Figura 02).

Foi observado também que nos anos de La Niña a precipitação foi superior a climatologia apenas $97,2 \mathrm{~mm}$ no período chuvoso e 4,5 $\mathrm{mm}$ na estação seca e no ano de ocorrência de El Niño a precipitação foi inferior 71,2 mm em relação a normal climatológica no período chuvoso e 35,1 na estação seca. O ano de 2016 teve a ocorrência de um El Niño forte segundo classificação do Centro de Previsão de Tempo e Estudos Climáticos (CPTEC, 2020), o que intensificou seus efeitos (Figura 02).

As temperaturas apresentaram médias sempre superiores no ano de El Niño em torno de $27,31^{\circ} \mathrm{C}( \pm 1,07)$ em comparação ao ano de La Niña $26,43^{\circ} \mathrm{C}( \pm 0,95)$ e da climatologia $26,39^{\circ} \mathrm{C}( \pm 0,80)$ (Figura 02$)$. Comportamento já esperado, devido o El Niño além da de reduzir o regime hídrico na região de Castanhal, tem como característica a elevação das temperaturas, ao contrário da La Niña que proporciona uma diminuição nas águas do pacífico que ocasiona em menores temperaturas e um maior índice pluviométrico dependendo da sua intensidade (FIORIN, 2015). 
Figura 02. Precipitação Pluviométrica (mm) e Temperatura do $\operatorname{Ar}\left({ }^{\circ} \mathrm{C}\right)$ em anos de El Niño (2011), La Niña (2016) e Climatologia (1989 - 2019).

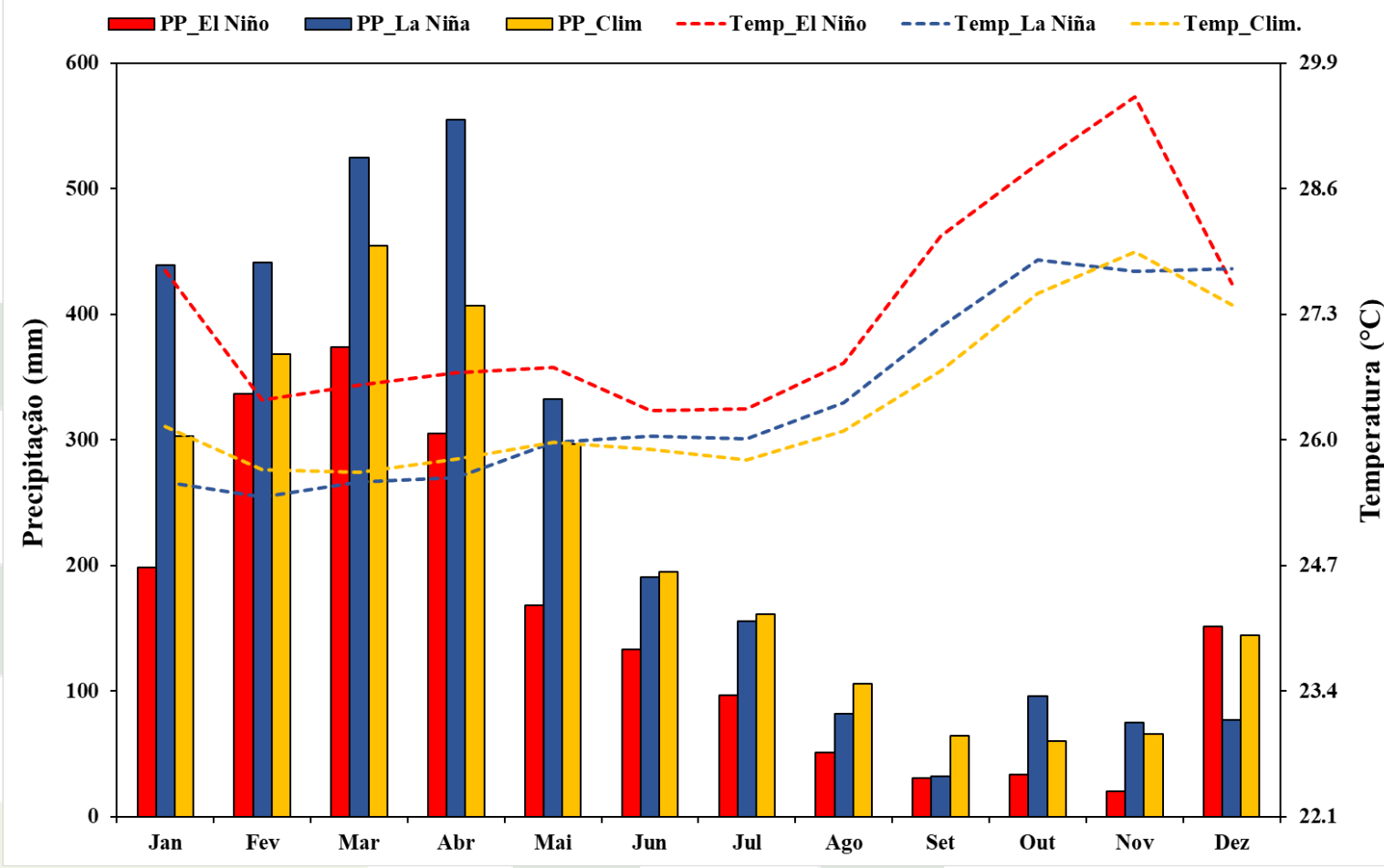

Fonte: Própria (2020).

A sazonalidade na precipitação são uns dos fatores que mais impactam a agricultura, principalmente as de sequeiro, no qual seu desempenho final depende inteiramente da distribuição do total de chuva (MARENGO et al, 2011), e de forma particular o regime pluviométrico na amazônia concentra seu maior volume durante as estações do verão e outono austral que corresponde aos meses de dezembro a maio e apresenta os menores volumes durante o período seco, correspondente aos meses de junho a Novembro (De Souza et al., 2017).

No caso na região de estudo as precipitações correspondentes aos meses chuvosos ( Dezembro a Agosto) correspondeu no ano de El Niño a 95,5\% do volume total anual, no de La Niña 93,2\% e na normal climatológica cerca de 92,8\% das chuvas. Na estação seca que na região corresponde aos meses de setembro, outubro e novembro, o somatório das chuvas representou apenas 4,5\% do volume total das chuvas no ano de El Niño, 6,8\% no de La Niña e 7,2\% na climatologia (Figura 02).

Nos meses de menores índices pliviométricos apresentam temperaturas mais elevadas (Figura 2) o que resulta maiores taxas de evapotranspiração (ETP) e maior défict hídrico e menores valores de evapotranspiração real de cultura (ETR) independente do ano, porém os ano de El Niño apresentou menores valores de ETR, tendo como destaque o mês de novembro 
com 24,9 mm que começou a aumentar com o ínicio das chuvas do período chuvoso, e maiores valores de ETP, principalmente no mês de outubro $24,9 \mathrm{~mm}$ que é o que possui menor total de chuvas 33,9 mm (Figura 03). Esse comportamento é explicado devido a grande demanda pela atmosfera nesse período com temperaturas mais altas e menor disponibilidade de água (JESUS, 2015).

Figura 03. Variação da evapotranspiração e evapotranspiração de referência em anos de El Niño (2011), La Niña (2016) e Climatologia (1989 - 2019).

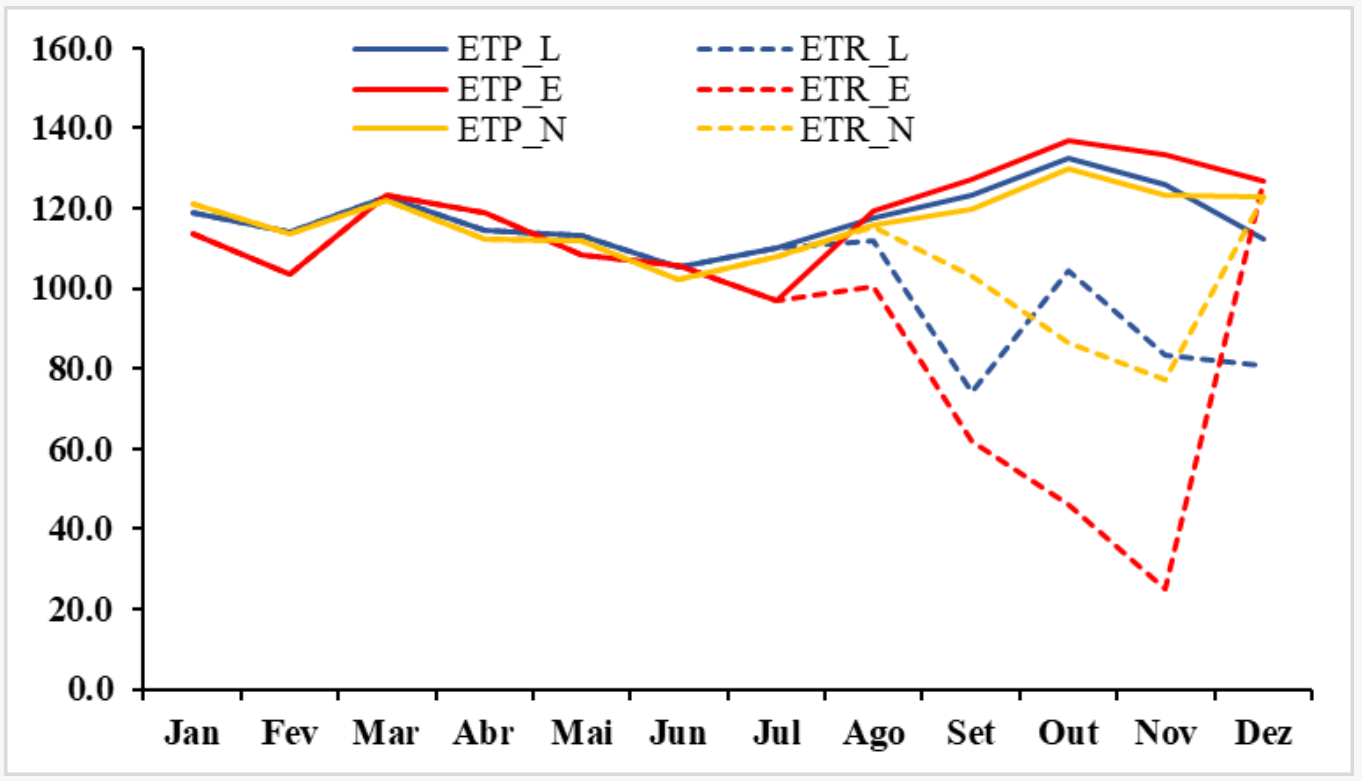

Fonte: Própria (2020).

Os meses de dezembro a agosto de maiores índices pluviométricos apresentam evapotranstranspiração potencial (ETP) e evapotranspiração (ETR) inferiores a precipitação, suprindo a demanda de água do solo no mês de dezembro e atingindo a capacidade de campo, e posteriormente a partir do mês de janeiro a julho apresentando excedente hídrico que totalizou 793,2 mm no ano de El Niño, 1791,2 no ano de La Niña e 1344,1 na climatologia (Figura 04). Esse tipo de monitoramento do armazenamento de água no solo auxilia servindo de indicador do estado hídrico do solo que possinilita as tomadas de decisão para amenizar os impactos negativos et al., 2011).

Essa retirada de água do solo entre os meses de agosto a novembro devido as condições de déficit hídrico resultante da má distribuição das chuvas, afeta diretamente o crescimento e desenvolvimento das plantas, necessitando de estratégias como a irrigação para a suprir a demanda de água da planta (OLIVEIRA et al., 2018). 
Figura 04. Extrato do Balanço hídrico mensal. Excedente (EXC) e Deficiência (DEF) em anos de El Niño (2011), La Niña (2016) e Climatologia (1989 - 2019).

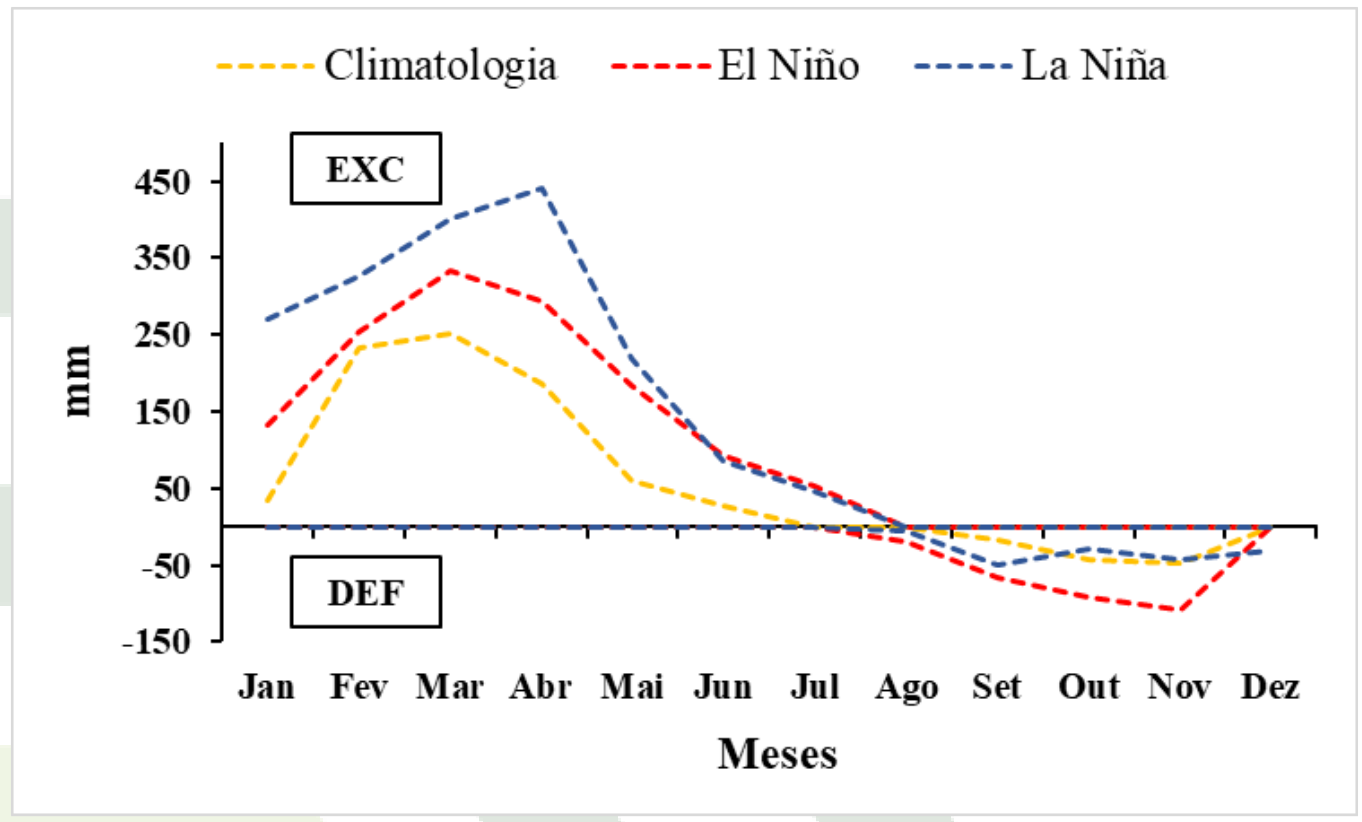

Fonte: Própria (2020).

A produção de açaí ao longo dos anos apresenta uma crescente, e as oscilações em alguns anos podem ser explicadas analisando o comportamento das variáveis climáticas. Especificamente no ano de ocorrencia de La Niña em 2011 a produção de açai em Castanhal cresceu cerca de 91,4\% (1552 T) a mais em relação ao ano de 2014, e no ano que ocorreu o El Nino a produção diminuiu 10\% (800 T) em relação ao ano de 2015 , redução que se repetiu no ano de 2017 com produção 10\% (800 T) menor em relação ao ano anterior, expressando efeitos do El Niño forte que ocorreu em 2016 (Figura 05).

A deficiência de água diminuia a produtividade e prolonga o período de sazonalidade (entresrafra) da produção de açaí (FARIAS NETO, et al. 2010). As palmeiras sob condições de déficit hídrico expressam seus efeitos negativos a curto, médio e o longo prazo, como BayonaRodriguez et al. (2019) apresentam em seu estudo com palma de óleo, onde observou aborto de cachos em plantas jovens, queda no peso médio dos cachos, aborto de inflorescências que pode perdurar por meses além da substituição na emissão de flores femeninas por masculinas, fatores que impactam na produção final da planta.

Em casos de deficiência hídrica duradouro, as plantas como a palma de óleo manifestam alterações em sua morfologia e em sua fisiologia e bioquímica que resultam em produção de fotoassimilados (CORLEY et al., 2018; VIANA et al.; 2019), além da diminuição na parte 
aérea e do sistema, e e alterações como o enlongamento, encurvamento em folhas precoces (MÉNDEZ et al. 2012).

A baixa produção de assimilados influencia diretamente no fruto, e essa diminuição ocorre pela menor taxa de assimilação de $\mathrm{CO} 2$ com o fechamento dos estômatos devido a perda de turgescência, maiores taxas de abscisão foliar, senescência e fotorespiração e consequentemente menores taxa de fotossítese (ZIVCAK et al., 2015).

Figura 05. Produção anual de frutos de açaí (em Ton.) em Castanhal - PA no período de 2000 a 2018)

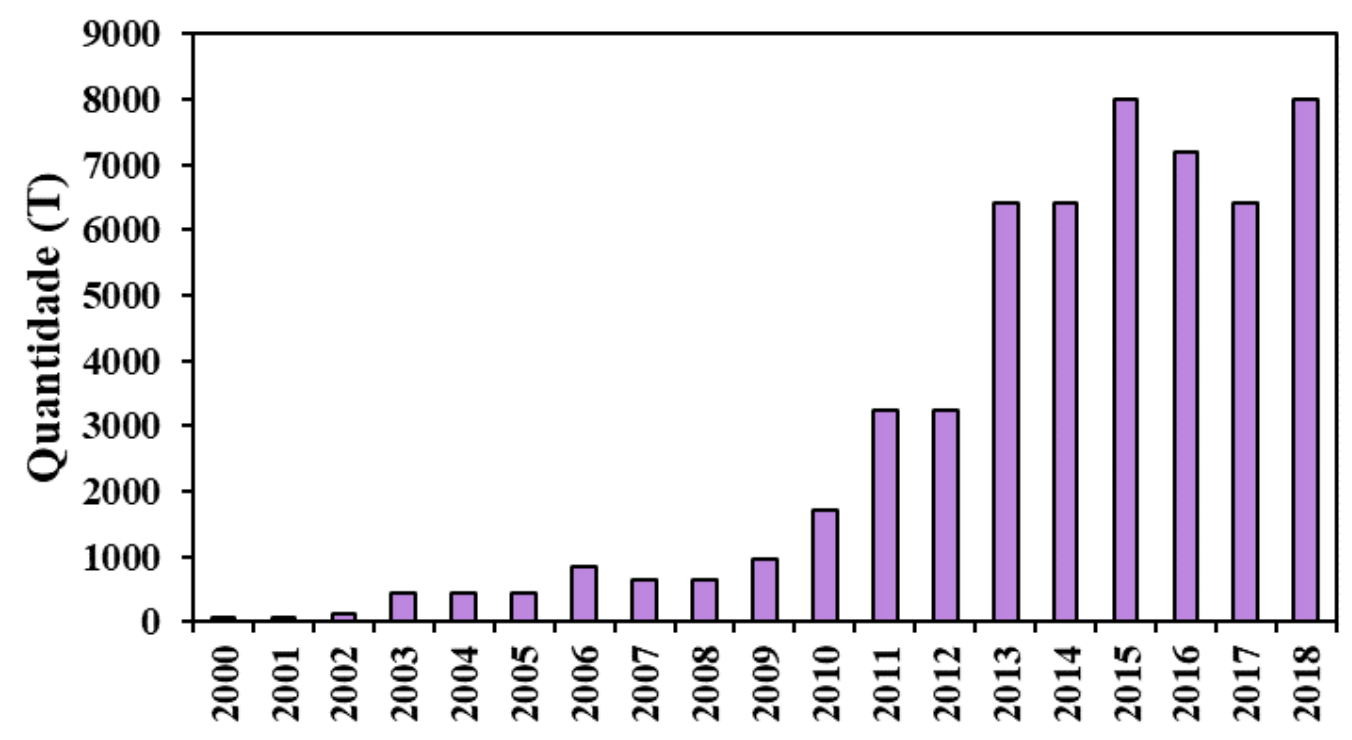

Anos

Fonte: IBGE (2020).

Esperava-se uma diminuição mais acentuada na produção no ano de ocorrência do El Niño, porém isso pode ser explicado devido o uso crescente da irrigação que segundo Farias Neto et al., (2010) além de assegurar a produção nos períodos de estiagem , também é uma garantia no aumento da produtividade. E a utilização da irrigação necessita de um planejamento e estratégias por partes dos produtores ( SIMÃO et al., 2015), além de conhecimentos relacionados a variabilidade climática do local, para determinação de um melhor manejo da cultura (Blain, 2009).

\section{CONCLUSÕES}

Os resultados demonstraram que a ocorrência de La Niña no município de Castanhal no ano de 2011 aumentou exponencialmente a produção de açaí, e de El Niño em 2016 diminui, porém apesar de ter sido classificado como forte o impacto na produção foi amenizado possivelmente com o uso da irrigação. A disponibilidade de água em Castanhal no período de 
janeiro a julho apresentaram grande volume de chuva e excedente hídrico, resultando em maiores produções independente da ocorrência ou não de ENOS. Já no período de estiagem (Agosto a Novembro) é recomendada a utilização da irrigação para suprir a demanda hídrica da cultura, principalmente nos anos de El Niño, onde os resultados apontaram maior déficit e diminuição na produção.

\section{REFERÊNCIAS}

ALVARES, C. A.; STAPE, J. L.; SENTELHAS, P. C.; MORAES GONÇALVES, J. L.; SPAROVEK, G. Köppen's climate classification map for Brazil. Meteorologische Zeitschrift, v. 22, n. 6, p. 711-728, 2013.

ARMANDO, D. M. S. Caracterização das batedeiras e portos de recebimento de açaí no estado do Amapá. 2015. 51f. Especialização (Especialização em Economia e Meio Ambiente) - Setor Ciências Agrárias, Universidade Federal do Paraná, Curitiba, 2015.

ASSAD, E.; PINTO, H. S. Aquecimento Global e a nova Geografia da Produção agrícola no Brasil. Brasília: Embrapa. 2008.

BARRETO, P. N.; SILVA R. B. C.; SOUZA, W. S.; COSTA, G. B.; NUNES, H. G. G. C.; SOUSA, B. S. B. Análise do balanço hídrico durante eventos extremos para áreas de floresta tropical de terra firme da Amazônia Oriental. In: XVI CONGRESSO BRASILEIRO DE AGROMETEOROLOGIA, Belo Horizonte. Anais... Belo Horizonte.2009.

BAYONA-RODRIGUEZ, C. J.; ROMERO, H. M. Physiological and agronomic behavior of commercial cultivars of oil palm (Elaeis guineensis) and OxG hybrids (Elaeis oleifera $\mathrm{x}$ Elaeis guineensis) at rainy and dry seasons. Australian Journal of Crop Science, v.13, n.03, p. 424$432,2019$.

BLAIN, G. C. Análises estatísticas das tendências de elevação nas séries anuais de temperatura mínima do ar no estado de São Paulo. Bragantina, Campinas, v.68, n.3, p.807-815, jul. /Set. 2009.

CPTEC, Centro de Previsão de Tempo e Estudos Climáticos. MONITORAMENTO DO EL NIÑO DURANTE DJF-2019. Disponível em: http://enos.cptec.inpe.br/. Acesso em: 18 de setembro de 2020.

CAMARGO, A. P. Balanço hídrico no Estado de São Paulo. Campinas: IAC, 28p. Boletim Técni-co, v. 116, 1971.

CARVALHO, P. H.; NETO, D. D.; TEODORO, F. E. R.; MELO. Balanço hídrico climatológico, armazenamento efetivo da água no solo e transpiração na cultura de café. Biosci. J., Uberlândia, v. 27, n. 2, p. 221-229, Mar./Apr. 2011.

COMPANHIA NACIONAL DE ABASTECIMENTO - CONAB. Proposta de Preços Mínimos / Companhia Nacional de Abastecimento - v.1 - (2015). - Brasília: Conab, 2015 v. Trimestral. Disponível em: http://www.conab.gov.br. Acesso em: 19 de setembro de 2020.

COUTINHO, M. D. L.; COSTA, M. S.; SILVA, A. R.; SANTOS, T. S.; GOMES, A. C. S.; 
MORAIS, M. D. C., SANTOS, P. V. Balanço hídrico mensal para dois municípios do estado da Paraíba. Ciência e Natura, Santa Maria, v. 37 n. p. 160-170.4 set-dez. 2015.

CORLEY, R.H.V.; RAO, V.; PRAIWAN, T. Breeding for drought tolerance in oil palm. Journal of Oil Palm Research, v. 30, n. 1, p. 26-35, 2018.

DE SOUZA, E. B.; DA SILVA FERREIRA, D. B.; GUIMARÃES, J. T. F.; DOS SANTOS FRANCO, V.; DE AZEVEDO, F. T. M.; DE MORAES, B. C. Padrões climatológicos e tendências da precipitação nos regimes chuvoso e seco da Amazônia oriental. Revista Brasileira de Climatologia, v. 21, 2017.

FARIAS NETO, J. T.; VASCONCELOS, M. A. M.; SILVA, F. C. F. Cultivo, Processamento, Padronização, e Comercialização do açaí na Amazônia. Fortaleza. Instituto Frutal, 2010.

FIORIN, T. T; ROSS, M. D. Climatologia agrícola, Santa Maria: Universidade Federal de Santa Maria, Colégio Politécnico. Rede e-Tec Brasil, 82 p. 2015.

GUIMARÃES, L.A.C. O açaí já "parou” o carioca? Estudo qualitativo do consumo da polpa de açaí na cidade do Rio de Janeiro. Belém: NAEA, 17p. (NAEA, Paper, 90). 1998.

HOMMA, A. K. O.; NOGUEIRA, O. L.; MENEZES, A. J. E. A.; CARVALHO, J. E. U.; NICOLI, C. M. L.; MATOS, G., B. Açaí: Novos desafios e tendencias. Amazônia: Ciência e Desenvolvimento, v. 1, n. 2, p. 7-23, 2006.

IBGE - Instituto Brasileiro de Geografia e Estatística. Pesquisa Pecuária Municipal. 2018. Disponível em: https://sidra.ibge.gov.br/pesquisa/ppm/quadros/brasil/2018. Acesso em: $19 \mathrm{de}$ setembro de 2020.

JESUS, J. B. Estimativa do balanço hídrico climatológico e classificação climática pelo método de Thornthwaite e Mather para o município de Aracaju- SE. Scientia Plena, v 11, n 5. 2015.

MARENGO, A. J.; ALVES, L. M.; BESERRA, A. E.; LACERDA, F. F. Variabilidade e mudanças climáticas no semiárido brasileiro. In: MEDEIROS, S. S.; GHEYI, H. R.; GALVÃO, C. O.; PAZ, V. P. S. Eds. Recursos hídricos em regiões áridas e Semiáridas. Campinas Grande PB: Instituto Nacional do Semiárido, 2011. p. 383-416.

MENEZES, F. P.; FERNANDES, L. L.; DA ROCHA, E. J. P. O uso da estatística para regionalização da precipitação no Estado do Pará, Brasil. Revista Brasileira de Climatologia, v. 16, 2015.

MÉNDEZ, Y.D.R.; CHACÓN, L.M.; BAYONA, C.J.; ROMERO, H.M. Physiological response of oil palm interspecific hybrids (Elaeis oleifera H.B.K. Cortes versus Elaeis guineensis Jacq.) to water déficit. Brazilian Journal of Plant Physiology, v. 24, n. 4, p. 273 $280,2012$.

NOAA. Nationat Oceanic and Atmospheric Administration. Disponível em: https://www.noaa.gov . Acesso em: 18 de setembro de 2020.

NOGUEIRA, A. K. M.; SANTANA, A. C.; GARCIA, W. S. A dinâmica do mercado de açaí fruto no Estado do Pará: de 1994 a 2009. Revista Ceres, Viçosa, v. 60, n. 3, p. 324-331, 2013. 
OLIVEIRA, M.S.P.; FARIAS NETO, J. T.; QUEIROZ, J. A. L. Cultivo e manejo do açaizeiro para produção de frutos. In: Embrapa Amazônia Oriental-Artigo em anais de congresso (ALICE). In: VI Encontro Amazônico de Agrárias, Belém, PA. Ufra, 2014.

OLIVEIRA, J.A.M.; OLIVEIRA, C.M.M. Balanço hídrico climatológico e classificação climática para o município de Arinos - MG. Revista Brasileira de Agricultura Irrigada, v. 12, n. 6, p. $3021-3027,2018$.

PEREIRA, A.R.; ANGELOCCI, L.R.; SENTELHAS, P.C. Meteorologia agrícola. Piracicaba: Universidade de São Paulo, 192p, 2007.

PEREIRA, M. D., Souza Filho, J. F. de; Moura, M. O. "Análise da pluviosidade na microrregião de Sapé, Paraíba e sua relação com a produção da cana-de-açúcar." Revista Geonorte v3(9): 910-921.2016.

PESSOA, J. D. C.; TEIXEIRA, G.H de A. Tecnologias para inovação nas cadeias euterpe., Brasília, DF: Embrapa, p. 343, 2012.

REICHARDT, K. A água em sistemas agrícolas. Barueri (SP): Manole, 1990

SEDAP. Secretaria de Estado de Desenvolvimento Agropecuário e da Pesca, 2018. PANORAMA AGRÍCOLA DO PARÁ 2010 / 2018. Disponível em: http://www.sedap.pa.gov.br/content/a\%C3\%A7a\%C3\%AD . Acesso em: 14 de setembro de 2020.

SIMÃO, A. H.; MATOS, A. P.; VASCONCELOS, J. A. R.; QUEIROZ, G. B. de; RAMAIO, K. A. Estimativa da demanda hídrica e manejo da irrigação na cultura do abacaxi no município de Conceição do Araguaia, Estado do Pará. In: VI Simposio Brasileiro da Cultura do Abacaxi, 2015, Conceição do Araguaia - PA. VI Simpósio Brasileiro da Cultura do Abacaxi Anais. v. 01. p. 172-178, 2015.

STACKHOUSE, P. W. Jr.; ZHANG, T.; WESTBERG, D.; BARNETT, A. J.; BRISTOW, T.; MACPHERSON, B.; HOELL, J. M. POWER release 8 (with GIS applications) methodology (data parameters, sources, \& validation) documentation date may 1, 2018 (all previous versions are obsolete) (data version 8.0.1), 2018.

THORNTHWAITE, C. W.; MATHER, J. R. The water balance publications in Climatology, 8 (1). DIT, Laboratory of climatology, Centerton, NJ, USA, 1955.

VIANA, J.L.; ZOLIN, C.A.; SILVA, V.Q.R.; SOUZA, A.P. Respostas fisiológicas e produtivas da palma de óleo irrigada em fase inicial de desenvolvimento. Irriga, n. 24, v. 2, p. 405-423, 2019.

ZIVCAK, M.; KALAJI, H. M.; SHAO H. N.; OLSOVSKA, K.; BRESTIC, M. Photosyntec próton and electron transport in wheat leaves under prolonged moderate drought stress.

Journal of Photochemistry and Photobiology, v. 137, p. 107-115, 2015. 